\title{
Influence of Low-Frequency Sonolysis on the Stability of Reactions Involving Associates
}

\author{
Tatiana P. Kulagina*, Lev P. Smirnov, Zoya S. Andrianova \\ Institute of Problems of Chemical Physics, Russian Academy of Sciences, Chernogolovka, Moscow, Russia \\ Email: *tan@icp.ac.ru
}

How to cite this paper: Kulagina, T.P., Smirnov, L.P. and Andrianova, Z.S. (2019) Influence of Low-Frequency Sonolysis on the Stability of Reactions Involving Associates. Journal of Applied Mathematics and Physics, 7, 2950-2957.

https://doi.org/10.4236/jamp.2019.712205

Received: October 30, 2019

Accepted: November 30, 2019

Published: December 3, 2019

Copyright $\odot 2019$ by author(s) and Scientific Research Publishing Inc. This work is licensed under the Creative Commons Attribution International License (CC BY 4.0).

http://creativecommons.org/licenses/by/4.0/

\section{Open Access}

\begin{abstract}
The influence of low-frequency sonolysis on the kinetics bimolecular liquid-phase reactions was studied with due regard for the association (dimers and trimers) of starting reagents. The mathematical modeling of chemical reactions that were described by nonlinear differential equations is performed. The steady states, the singular points characteristics, the nature of concentration oscillations in the reaction system are described. With increasing frequency and amplitude of low-frequency sonic waves (up to some critical value), we observed the cessation of the reaction. This observation offers an additional tool for controlling reaction rate by the external action of low-frequency vibrations. The conclusions of the work are obtained under certain assumptions. The exact determination of the critical conditions for changes in dynamics is beyond the scope of the problem.
\end{abstract}

\section{Keywords}

Chemical Reaction, Kinetics, Liquid Medium, Reagent Association,

Low-Frequency Action, Bistability Regime, Oscillation, Resonant Change

\section{Introduction}

When studying the kinetics of liquid-phase reactions, the dependence of the rate constants on the concentration of the reagent, the extreme nature of the Arrhenius dependence of the rate constant on temperature were found [1] [2]. These kinetic anomalies are not described by the kinetics of a homogeneous medium. The character of these anomalies is most strictly explained by the concept of the structural organization of the liquid during the formation of associates due to intermolecular interactions [2] [3] [4]. Theoretical and experimental data show that the association of molecules affects the reactivity of the molecules that form the associates [3] [4] [5] [6]. 
The associative structure of a liquid reaction system opens up new possibilities for controlling the kinetics of reactions by various kinds of external influences. Although many works are devoted to the mechanical effect on liquid-phase reactions, they usually study the phenomenological aspects of the effect of high-frequency acoustic fields on the reaction system [7] [8].

Data on the effect of the association of reagents on the kinetics of reactions are still ignored in the literature, and the theory of phenomena induced by periodic mechanical action, taking into account the supramolecular structure of the reaction medium, is being developed relatively recently.

Previously, we have proposed [2] [3] [4] that some anomalies in the kinetics of bimolecular liquid-phase reactions can be explained by the formation of the associates of starting reagents. Earlier, the effect of sonolysis on the reaction kinetics in liquids was explored mostly for high-frequency acoustic waves [7] [8]. Meanwhile, noticeable chemical effects were also reported for low-frequency (10 - $100 \mathrm{~Hz}$ ) acoustic waves [9] [10] [11]. Deeper insight into the mechanism of this phenomenon can be reached via mathematical modeling of such reactions [12] [13]. The review aims at discussing possible approaches to regulate the kinetics of chemical reactions under the action low-frequency external action.

\section{Mathematical Model}

In this work, we modeled the influence of low-frequency sonolysis on the stability of intermediates in the $\mathrm{A}+\mathrm{B} \rightarrow \mathrm{C}$ reactions in associated liquids [2], by using the reaction of alcohol $\mathrm{A}$ and isocyanate $\mathrm{B}$ yielding urethane $\mathrm{C}$ as an example [1] with the formation of associates of reactant $A$, namely, dimer $A_{2}$ and trimer $A_{3}$.

The association of the reactants even a formally one stage bimolecular reaction turns into a multistage process:

$$
\begin{gathered}
\mathrm{A}+\mathrm{A} \leftrightarrow \mathrm{A}_{2} \quad\left(k_{1}, k_{-1}\right) \\
\mathrm{A}+\mathrm{A}_{2} \leftrightarrow \mathrm{A}_{3} \quad\left(k_{2}, k_{-2}\right) \\
\mathrm{A}+\mathrm{A}_{2}+\mathrm{A}_{3} \rightarrow 2 \mathrm{~A}_{3} \quad\left(k_{3}\right) \\
\mathrm{B}+\mathrm{B} \leftrightarrow \mathrm{B}_{2}\left(k_{4}, k_{-4}\right) \\
\mathrm{B}_{2}+\mathrm{A}_{3} \rightarrow \mathrm{C}+\mathrm{B}+2 \mathrm{~A} \quad\left(k_{5}\right) .
\end{gathered}
$$

Here, $[\mathrm{A}],\left[\mathrm{A}_{2}\right]$ and $\left[\mathrm{A}_{3}\right]$ are the concentrations of monomer, dimer and trimer forms of alcoyol, respectively, $[\mathrm{B}]$ is the concentration of isocyanate, $k_{i}$ and $k_{-i}$ are the rate constants for the forward $(i)$ and reverse $(-i)$ reactions, respectively.

The kinetic equations for the intermediates of the system under consideration have the form:

$$
\begin{aligned}
\mathrm{d}\left[\mathrm{A}_{2}\right] / \mathrm{d} t= & k_{1}[\mathrm{~A}]^{2}-k(v)_{-1}\left[\mathrm{~A}_{2}\right]-k_{2}[\mathrm{~A}]\left[\mathrm{A}_{2}\right]+k(v)_{-2}\left[\mathrm{~A}_{3}\right] \\
& -k_{3}[\mathrm{~A}]\left[\mathrm{A}_{2}\right]\left[\mathrm{A}_{3}\right] \\
\mathrm{d}\left[\mathrm{A}_{3}\right] / \mathrm{d} t= & k_{2}[\mathrm{~A}]\left[\mathrm{A}_{2}\right]+k_{3}[\mathrm{~A}]\left[\mathrm{A}_{2}\right]\left[\mathrm{A}_{3}\right]-k(v)_{-2}\left[\mathrm{~A}_{3}\right] \\
& -k_{4} k_{5}[\mathrm{~B}]^{2}\left[\mathrm{~A}_{3}\right]\left\{k_{-4}+k_{5}\left[\mathrm{~A}_{3}\right]\right\}^{-1}
\end{aligned}
$$


According to the ideas of A. Tobolsky and G. Eyring, associates are fragile formations that are easily destroyed by a mechanical field [14].

The external action with $\omega=2 \pi v$ generates tensile stress $\sigma$ that affects reaction rate constant

$$
k(\omega)_{-i}=k_{-0 i} \exp \left[\left(-E_{-i}+\gamma_{i} \sigma\right) / R T\right],
$$

where $\gamma_{i}$ is a structure-sensitive coefficient and $\sigma=P_{0} \sin (\omega t)$. Under steady-state conditions, the concentration of dimer $\mathrm{B}_{2}$ and intermediates is described by the following system of parametric equations:

$$
\begin{gathered}
\mathrm{d} x / \mathrm{d} t=\mu-a_{1} x \exp \left[\gamma_{1} P \sin (\omega t)\right]-a_{2} x+b y \exp \left[\gamma_{2} P \sin (\omega t)\right]-u x y \\
\mathrm{~d} y / \mathrm{d} t=a x-b y \exp \left[\gamma_{2} P \sin (\omega t)\right]+u x y-m y(g+y)^{-1},
\end{gathered}
$$

where $x=\left[\mathrm{A}_{2}\right], y=\left[\mathrm{A}_{3}\right], \mu=k_{1}[\mathrm{~A}]^{2}, a_{1}=k_{-1}, a_{2}=k_{2}[\mathrm{~A}], b=k_{-2}, u=k_{3}[\mathrm{~A}], m=$ $k_{4}[\mathrm{~B}]^{2}, g=k_{-4} k_{5}^{-1}$.

We also assume that a change in $k(v)$ during compression can be neglected in view of low compressibility of liquids. Moreover, the associates can be formed in case of some favorable mutual disposition of the reagents, which is infringed due to diffusion and formation of voids under the action of tension strain. With increasing separation between the reactive species, the reaction may transfer to a mode of diffusion control. Slow association at the stage of compression is known in the literature [5].

In order to escape cumbersome calculations, we solved the problem for a situation when $[\mathrm{A}]=[\mathrm{B}]=$ const, In this case, the expression for rate $w$ for formation of product $\mathrm{C}$ can be represented [3] [4] as:

$$
w=k_{4} k_{5}[\mathrm{~B}]^{2}\left[\mathrm{~A}_{3}\right]\left\{k_{-4}+k_{5}\left[\mathrm{~A}_{3}\right]\right\}^{-1}=m y(g+y)^{-1} .
$$

Accordingly, the expression for effective rate constant $\left(k_{\text {eff }}\right)$ acquires the form:

$$
k_{\text {eff }}=k_{4} k_{5}\left\{k_{-4}+k_{5}\left[\mathrm{~A}_{3}\right]\right\}^{-1}\left[\mathrm{~A}_{3}\right][\mathrm{A}]^{-1}[\mathrm{~B}] .
$$

It follows that the dependence of $w$ on $\omega$ manifests itself in an inexplicit way.

Equations (7) describes the dynamic behavior of a system with two degrees of freedom. The following situations are possible in terms of the concentrations of intermediates $\mathrm{A}_{2}$ and $\mathrm{A}_{3}$ : the absence of a steady-state solution, the existence of only one steady state, and, finally, the existence of two steady states. Solving system of Equations (7) makes it possible to obtain the kinetic concentration dependence for $x(t)$ and $y(t)$ and to study the process of reaching a stationary (equilibrium) mode.

Let us introduce the following notation:

$$
\begin{aligned}
& \mathrm{d} x / \mathrm{d} t=\Phi(x, y) \\
& \mathrm{d} y / \mathrm{d} t=\Psi(x, y)
\end{aligned} .
$$

Under the condition that $\Phi(x, y)=\Psi(x, y)=0$, let us find a singular point (an equilibrium point) $\left(x_{0}, y_{0}\right)$ in the $x, y$ phase plane from the equation

$$
\mathrm{d} y / \mathrm{d} t=\Psi(x, y) / \Phi(x, y),
$$


and consider changes in the integral curves $x(t)$ and $y(t)$ as a motion along the phase plane. In this case, the problem of finding the properties of the singular points reduced to studying the stability of the motion at the point $\left(x_{0}, y_{0}\right)$ using a known technique [1] [15].

For this purpose, consider Equation (9) with respect to the variables $(\xi, \eta)$ by representing the variables $x$ and $y$ in the following forms:

$$
\begin{aligned}
& x=x_{0}+\eta \\
& y=y_{0}+\xi
\end{aligned} .
$$

By substituting Equations (11) into Equations (9) and expanding the functions $\Phi(x, y)$ and $\Psi(x, y)$ into series, we obtain, in the first order of smallness, a linearized system:

$$
\begin{aligned}
& \mathrm{d} \eta / \mathrm{d} t=\Phi_{x} \eta+\Phi_{y} \xi \\
& \mathrm{d} \xi / \mathrm{d} t=\Psi_{x} \eta+\Psi_{y} \xi
\end{aligned},
$$

in which $\Phi_{x}, \Phi_{y}, \Psi_{x}, \Psi_{y}$ are values of the partial derivatives of the functions $\Phi$ $(x, y)$ and $\Psi(x, y)$ by $x$ and $y$, respectively, at the singular point $\left(x_{0}, y_{0}\right)$. The behavior of the solutions of system Equations (12) near the considered singular point is determined by the properties of the system of linear differential equations with constant coefficients.

The standard solution (12) can be represented as a superposition of exponentials:

$$
\begin{aligned}
& \eta=\Phi_{x} \mathrm{e}^{\delta_{1} t}+\Phi_{y} \mathrm{e}^{\delta_{2} t}, \\
& \xi=\Psi_{x} \mathrm{e}^{\delta_{1} t}+\Psi_{y} \mathrm{e}^{\delta_{2} t},
\end{aligned}
$$

where $\delta_{i}(i=1,2)$ are the roots of the characteristic equation

$$
\delta^{2}+p \delta+q=0,
$$

in which $p=\Phi_{x}+\Psi_{y}, q=\Phi_{x} \Psi_{y}+\Phi_{y} \Psi_{x}$ and. In this case, the stability of the steady-state solution is determined by the coefficients $p$ and $q$ of Equations (13).

\section{Results and Discussion}

The problem was numerically solved for the case $[\mathrm{A}]=[\mathrm{B}]=$ const (open system) by varying all the parameters of the system of Equations (7). Calculation below was carried out for model systems with the following parameters: $\left[\mathrm{A}_{2}\right]_{0}=$ $x(0)=1.8 \mathrm{M}$, and $\left[\mathrm{A}_{3}\right]_{0}=y(0)=0.23 \mathrm{M}, \mu=35 \mathrm{M} \cdot \mathrm{s}^{-1}$ and $m=200 \mathrm{M} \cdot \mathrm{s}^{-1}$.

For the case without external action $\left(\gamma_{1}=\gamma_{2}=0\right)$ the phase portrait of the system was determined by solving equation (10) and the linearized system of Equations (12) and (13). On the phase plane, the integral curves $x(t)$ and $y(t)$ (Figure 1) have the form of concentric circles emerging from a singular point at $x_{0}=2.51081$ and $y_{0}=0.33924$, which is a stable center.

The concentrations $x(t)$ and $y(t)$ were calculated with the same parameters from the system of Equation (7). Unusual oscillations are observed in the system the maximum of the amplitudes changes harmonically (as is typical for a stable central mode) with a rather long oscillation period (Figure 2). However, 


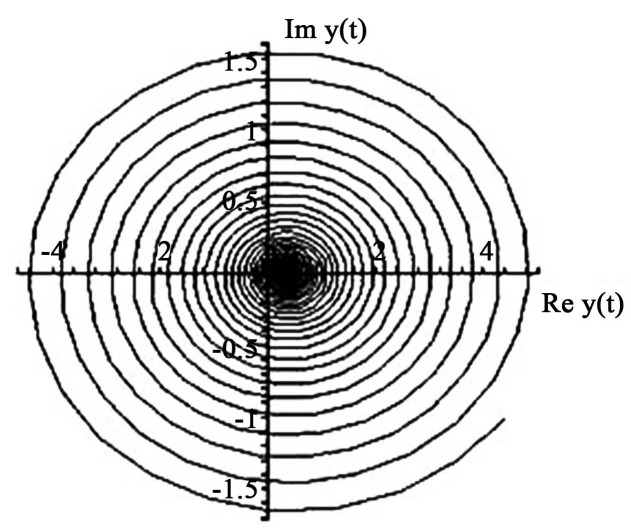

Figure 1. Phase portrait of $y=\left[\mathrm{A}_{3}\right]$ in neighborhood of singular point.

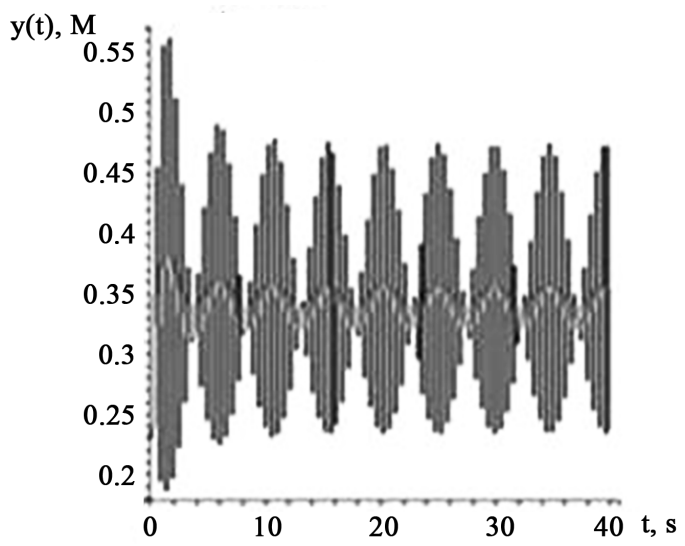

Figure 2. Dependence of concentrations $y=\left[\mathrm{A}_{3}\right]$ on time $t$ at $P=0$.

within each individual period of harmonic oscillations, there is a fine structure that is formed by oscillations with a smaller period.

The concentrations $x(t)$ and $y(t)$ were calculated from (7) for different amplitudes $P$ and frequencies $\omega$ and exhibited similar behavior. Essential change in the structure of oscillations happens at $\omega_{0}=0.4 \mathrm{~s}^{-1}$ and for $P>2$. With increasing $\omega$, functions $x(t)$ and $y(t)$ acquire rather complicate profiles (Figure 3 ) or exceedingly large spread (Figure 4 ), which can be explained by the presence $\left[\mathrm{A}_{3}\right]$ and high formation rate for associates $\mathrm{B}_{2}$. Increase in $\omega$ leads to the formation of unstable focuses.

For $[\mathrm{A}]=[\mathrm{B}]=$ const, we obtained the kinetics of change in the concentrations of associates $A_{2}$ and $A_{3}$ as a function of amplitude Pand frequency $\omega$ at starting stable-node stability. Two critical $\omega$ values were found to occur. For low $P$ and $\omega$, we observed oscillations in the concentration of the associates without change in the type of equilibrium. An increase in $P$ and/or $\omega$ results in hybrid oscillations. For low $\omega, P_{\min }$ is close to zero. With increasing, we come to a second critical phenomenon: bifurcation of the system state. At the bifurcation point, the oscillations are insignificant but, with increasing $\omega$, two kinds of harmonic oscillations with close periods but different amplitudes and concomitant pulsations come into being (see Figure 3). 


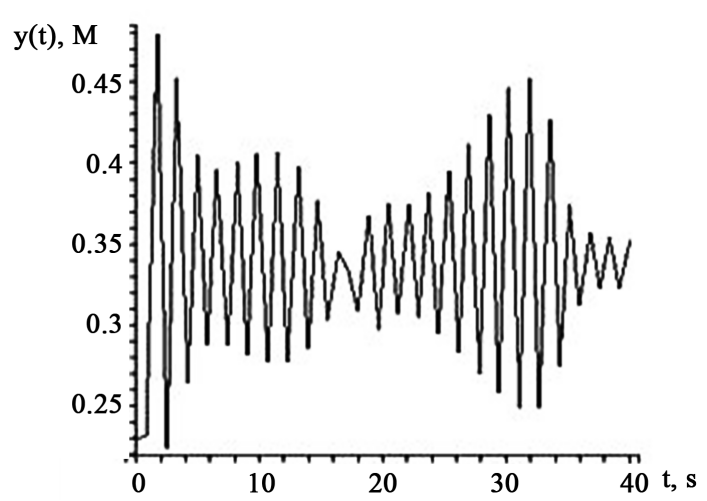

Figure 3. Dependence of concentrations $y=\left[\mathrm{A}_{3}\right]$ on time $t$ at $P=3$.

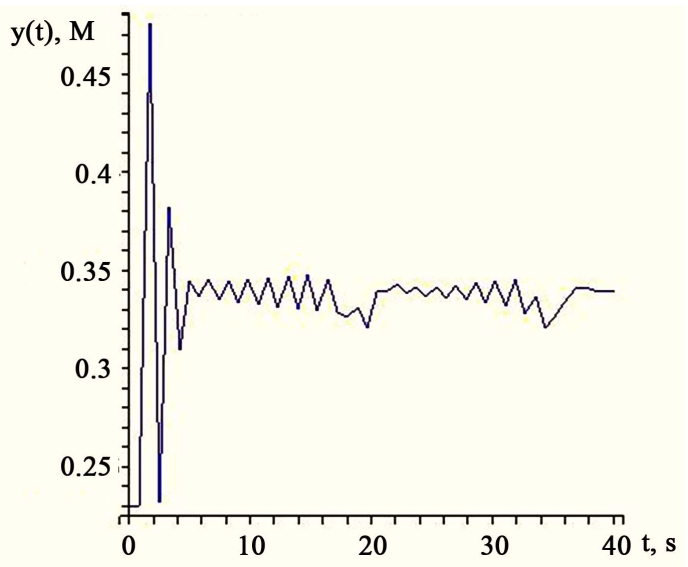

Figure 4. Dependence of concentrations $y=\left[\mathrm{A}_{3}\right]$ on time $t$ at $P=4$.

A reasonable question arises on the applicability of the results obtained for open systems to the closed ones which are most frequent in experimental. Strictly speaking, reagent concentrations in closed systems remain unsteady only during a certain period of reaction during which the concentrations of intermediates may be considered as quasi-stationary. It has been shown experimentally that the dissipation structures can also form in closed systems as well [14].

\section{Conclusions}

As far as we know, this work made almost the first attempt to generalize data on the kinetics of a complex process in a liquid medium during the association of reagents.

The above results suggest that sonolysis can be used as an additional tool for controlling the liquid-phase reactions involving the associates of starting reagents. The development of ideas about the structural organization of reagents in the liquid phase, about its effect on chemical reactions, about changes in the structural organization of reagents due to external influences of a different nature, allows a new approach to solve applied problems of chemistry, biochemistry, and medicine. 


\section{Acknowledgements}

This work was financially supported by FASO (grant No. 0089-2019-0002).

\section{Conflicts of Interest}

The authors declare no conflicts of interest regarding the publication of this paper.

\section{References}

[1] Tiger, R.P., Tarasov, D.N. and Entelis, S.G. (1996) Use of Scaling Approach to the Study of Kinetics and Mechanism of Molecular Reactions in Associated Media. Khimicheskaya Fizika, 15, 11-19.

[2] Smirnov, L.P. and Kulagina, T.P. (2017) Features of the Kinetics of Chemical Reactions in a Nanostructured Liquid. Russian Journal of Physical Chemistry B, 11, 786-797. https://doi.org/10.1134/S1990793117050207

[3] Kulagina, T.P., Smirnov, L.P. and Andrianova, Z.S. (2017) Kinetics of a Bimolecular Chemical Reaction in the Liquid Phase with Association of Both Reactants. Doklady Physical Chemistry, 475, 126-128. https://doi.org/10.1134/S0012501617070065

[4] Kulagina, T.P., Andrianova, Z.S. and Smirnov, L.P. (2018) Specific Features of a Two-Phase Bimolecular Chemical Reaction in the Case of the Association of Both Reactants. Kinetics and Catalysis, 59, 17-22. https://doi.org/10.1134/S0023158418010056

[5] Nicolis, G. and Prigogine, I.P. (1977) Self-Organization in Non-Equilibrium Systems. Wiley-Interscience, New York.

[6] Kononov, L.O. (2015) Chemical Reactivity and Solution Structure: On the Way to a Paradigm Shift? RSC Advances, 5, 46718-46734.

https://doi.org/10.1039/C4RA17257D

[7] Price, G.J. (1999) Polymer Sonochemistry Controlling the Structure and Properties of Macromolecules. In: Crum, L.A., Mason, T.J., Reisse, J.L. and Suslick, K.S., Eds., Sonochemistry and Sonoluminescence, NATO ASI Series, Kluwer Academic Publishers, Amsterdam.

[8] Margulis, MA. (1986) Sonochemical Reactions and Sonoluminescence. Khimiya, Moscow. (In Russian)

[9] Khosin, V.G. (2004) Strengthening Epoxy Polymers. Dom Pechati, Kazan. (In Russian)

[10] Ganiev, R.F., Fomin, V.N., Malyukova, E.B., et al. (2005) Wave Treatment of Latex-Based Disperse Systems. Doklady Chemistry, 403, 164-167. https://doi.org/10.1007/s10631-005-0062-x

[11] Mingaleev, N.Z., Zinnurov, Z.G., Kostina, L.E., et al. (2006) Polyurethane on the Basis of Polyair Subjected to Acoustic Processing. Kauch Rezina, 5, 16-18. (In Russian)

[12] Smirnov, L.P., Kulagina, T.P. and Andrianova, Z.S. (2017) Influence of Low-Frequency Vibrations on the Chemical Reaction Rate in the Liquid Phase for Reagent Association. Russian Chemical Bulletin, 66, 30-33. https://doi.org/10.1007/s11172-017-1695-x

[13] Kulagina, T.P., Smirnov, L.P. and Andrianova, Z.S.T.P. (2018) Mathematical Simulation of Low-Frequency Mechanical Action on Bimolecular Reaction Kinetics in a Structured Liquid. Russian Journal of Physical Chemistry B, 12, 657-662. 


\section{https://doi.org/10.1134/S1990793118040139}

[14] Tobolsky, A. and Eyring, H. (1943) Mechanical Properties of Polymer Materials. The Journal of Chemical Physics, 11, 125-134. https://doi.org/10.1063/1.1723812

[15] Ebeling, W. (1976) Structurbildung bei irreversiblen Prozessen. Leipzig. Teubner. 